\title{
INTERVENÇÃO FONOAUDIOLÓGICA NA SÍNDROME DE KABUKI: RELATO DE CASO
}

\section{Language intervention in the Kabuki syndrome: case report}

\author{
Maria Cláudia Brito ${ }^{(1)}$, Andréa Regina Nunes Misquiatti (2)
}

\begin{abstract}
RESUMO
Tema: este trabalho teve como objetivo descrever o processo de intervenção fonoaudiológica em um caso de síndrome de Kabuki, com ênfase na terapia de linguagem. Procedimentos: trata-se de relato de caso de uma criança de seis anos e seis meses de idade, gênero feminino, com diagnóstico de síndrome de Kabuki, que realizou terapia fonoaudiológica durante três anos em uma clínica-escola. Foi realizada análise documental dos dados do prontuário relativos à anamnese, avaliação e relatórios terapêuticos fonoaudiológicos, exames e avaliações multidisciplinares. Aos três anos e cinco meses, a criança passou por avaliação fonoaudiológica e apresentou, como histórico, características típicas da síndrome de Kabuki: alteração cardíaca, episódios de pneumonia, otite média secretora recorrente, alterações ortopédicas e crises convulsivas. Na avaliação fonoaudiológica, apresentou tempo de atenção auditiva e visual reduzido, dificuldade de compreensão oral, comunicação por meio de poucos gestos e vocalizações e problemas de comportamento. Resultados: o processo terapêutico teve como objetivo principal promover o desenvolvimento da linguagem com ênfase na interação social por meio de atividades lúdicas, orientação familiar e escolar. Foi observada melhora na compreensão e emissão oral, no contato social e manutenção da atenção. Conclusão: este estudo permitiu divulgar o percurso de atuação fonoaudiológica na síndrome de Kabuki, do processo diagnóstico à terapia de linguagem. Verificou-se que a terapia foi eficaz no que se refere às habilidades comunicativas e de interação social. Esses dados podem contribuir para elucidar lacunas existentes na literatura acerca da terapia fonoaudiológica na síndrome em questão e subsidiar intervenções clínicas nesses casos.
\end{abstract}

DESCRITORES: Genética; Linguagem; Avaliação; Reabilitação

\section{INTRODUÇÃO}

A síndrome de Kabuki (SK) é um distúrbio congênito raro ${ }^{1-4}$, tendo sido relatados cerca de 350 casos na literatura ${ }^{5}$. Foi descrita no Japão por Niikawa e Kuroki em 1981, de forma independente e concomitante, por isso é também chamada de síndrome Niikawa-Kuroki ${ }^{1}$. Foi denominada síndrome da Maquiagem de Kabuki - SMK (Kabuki make-up syndrome), pois a facies típica

(1) Fonoaudióloga; Mestre em Psicologia do Desenvolvimento e Aprendizagem pela Universidade Estadual Paulista; Doutoranda em Educação na Universidade Estadual Paulista.

(2) Fonoaudióloga; Professora do Departamento de Fonoaudiologia da Universidade Estadual Paulista, UNESP, Marília, SP; Doutora em Linguística pela Universidade de São Paulo.

Conflito de interesses: inexistente assemelha-se à maquiagem do tradicional teatro japonês de Kabuki ${ }^{6}$, no entanto, o termo "maquiagem" não tem sido mais utilizado.

A prevalência da síndrome no Japão foi estimada em 1:32000 em todo o mundo, um número crescente de pacientes tem sido diagnosticado e não há predileção por sexo ou raça ${ }^{7,8}$.

Quanto à etiologia, foi relatada uma $8 p$ duplicação no cromossomo 8p22-8p23.1 entre seis pacientes com SK ${ }^{9}$. Todavia, outros estudos não confirmaram esses achados ${ }^{1,3}$. Outra pesquisa investigou 10 pacientes e afirmou que é possível encontrar heterogeneidade genética entre indivíduos com a síndrome e que seria improvável que todos tenham uma $8 p$ duplicação intersticial ${ }^{4}$, sendo que algumas pesquisas descreveram outros marcadores genéti$\cos ^{1,10,11}$. Em 2008, foi publicado um trabalho pelos mesmos autores do primeiro estudo, reanalisando os dados do estudo anterior, que assinalou que não 
há uma 8p duplicação no cromossomo 8p22-8p23.1 em todos os indivíduos com SK, sendo necessárias mais pesquisas para esclarecer sua etiologia ${ }^{12}$.

O fenótipo clínico observado é muito variável e há cinco características fundamentais, reconhecidas como "Pêntade de Niikawa": 1. Face dismórfica, encontrada em $100 \%$ dos casos; 2 . Anomalias esqueléticas, incluindo braquidactilia do $5^{\circ}$ dedo e/ ou deformação vertebral (92\%); 3. Alterações dermatoglíficas, com polpa digital abaulada e alças digitais ulnares $(93 \%) ; 4$. Leve a moderado retardo mental $(92 \%)$ e 5 . Retardo do crescimento pósnatal $(83 \%)^{6}$.

Foi observada também a ocorrência de perda auditiva, que pode ser parcial ou total, dependendo do grau de anormalidade do ouvido interno ${ }^{13}$, de retardo mental ${ }^{6,10}$ e psicomotor ${ }^{6}$, e o grau de acometimento mostrou-se bastante variável, oscilando de normal a leve $(\mathrm{Ql}=90)$ ou a grave atraso do desenvolvimento mental $(\mathrm{QI}=15$ a 20$)$, sendo que a maioria dos pacientes enquadra-se na faixa moderada a grave ${ }^{14}$.

No que tange aos aspectos da comunicação, desde 1981 foram relatados mais de 300 casos em diversos países, mas um número limitado desses estudos abordou o desenvolvimento de fala e linguagem ${ }^{8}$. Pouco se sabe acerca das habilidades intelectuais e do comportamento desses indivíduos, além do fato de que a maioria apresenta deficiência mental ${ }^{15}$, mas um número crescente de estudos confirmou que alterações na comunicação são comuns ${ }^{16}$.

O desenvolvimento da linguagem pode estar atrasado, com alterações nas habilidades expressivas e receptivas ${ }^{8,17-20}$. Foram encontradas habilidades morfossintáticas restritas, dificuldades lexicais e pragmáticas ${ }^{8}$. Embora o vocabulário e habilidades gramaticais possam estar reduzidos, algumas crianças apresentaram vocabulário adequado à idade ${ }^{21}$. Em alguns casos, não foi observado desenvolvimento de fala significativo com idade de 10 anos ${ }^{19} \mathrm{e}$, em outros, há um atraso de fala moderado ${ }^{21}$. Foram observadas alterações na voz 21, disartria, dispraxia de fala e alterações na articulação dos sons, relacionados à hipotonia oral ${ }^{19}$.

Foram descritos graus variáveis de deficiência mental, dificuldades de aprendizagem ${ }^{15}$, comportamento adaptativo deficiente, déficits de atenção, hiperatividade, impulsividade, comportamentos obsessivos e ansiedade ${ }^{15}$. Alguns autores observaram crianças comunicativas, amigáveis e socialmente adaptadas ${ }^{21}$. No entanto, dificuldades de interação e comportamentos autísticos também foram referidos ${ }^{14,17,22}$. Além disso, excelente memória de longo prazo, contato visual restrito ${ }^{23}$ e hiperlexia ${ }^{17}$ foram relatados.
Em relação à literatura nacional, poucos estudos foram realizados, sendo que foi relatado um caso com alteração auditiva e atraso no desenvolvimento da linguagem ${ }^{7}$ e uma paciente com comportamentos autísticos, como movimentos estereotipados e repetitivos e ausência de resposta a estímulos externos ${ }^{14}$.

A partir da revisão de literatura, notou-se o restrito número de estudos acerca de aspectos fonoaudiológicos e especialmente de procedimentos terapêuticos empregados na SK. Dessa forma, este estudo teve como objetivo descrever o processo de intervenção fonoaudiológica em um caso de síndrome de Kabuki, com ênfase na terapia de linguagem.

\section{APRESENTAÇÃO DO CASO}

Este estudo baseia-se no relato de caso de uma criança de seis anos e seis meses de idade, do gênero feminino, filha única de uma família de classe média baixa, ambos os pais com $2^{\circ}$ grau do ensino médio completo, que realizou atendimento fonoaudiológico em uma clínica-escola durante três anos.

Para elaboração deste trabalho, foi realizada análise documental dos dados do prontuário referentes à anamnese, avaliação e relatórios terapêuticos fonoaudiológicos, além de exames e avaliações multidisciplinares.

A anamnese e avaliação fonoaudiológica permitiram a obtenção de dados sobre o desenvolvimento neuropsicomotor, comunicação e cognição, bem como as dinâmicas familiar e escolar da criança. As avaliações multidisciplinares incluíram: avaliação genética clínica e neurológica, otorrinolaringológica e audiológica.

Quanto ao processo terapêutico, a cada semestre eram elaborados relatórios referentes às sessões de terapia, o que possibilitou verificar tal percurso e suas evoluções.

Este estudo foi autorizado mediante assinatura do Termo de Consentimento Livre e Esclarecido elaborado para fins específicos desta pesquisa, segundo a Resolução №. 196/96 e teve aprovação do Comitê de Ética em Pesquisa (CEP) da Faculdade de Filosofia e Ciências de Marília - Universidade Estadual Paulista (UNESP) - São Paulo, processo $\mathrm{n}^{\circ}$. 1275-2007.

\section{Dados de anamnese}

A mãe procurou a clínica-escola com queixa de atraso no desenvolvimento de fala quando a criança tinha três anos e cinco meses de idade. Segundo ela, a criança apresentou alteração cardíaca congênita, icterícia neonatal e pés e mãos edemaciados. 
Aos oito dias de vida, foi verificado sopro no coração; com um mês e 15 dias, a criança foi internada por 18 dias em UTI devido ao quadro de pneumonia. A criança apresentou perfuração de membrana timpânica no ouvido esquerdo e otite média secretora recorrente bilateral aos quatros meses de idade; quadros de pneumonia de repetição de três em três meses; lacrimejamento e secreção ocular. Aos dois anos de idade, iniciou tratamento ortopédico devido a alterações da coluna vertebral e, com dois anos e quatro meses, realizou intervenção cirúrgica para desobstrução dos ductos lacrimais. Com dois anos e nove meses de idade, apresentou a primeira crise convulsiva e um outro episódio sem data definida. Realizava eletrocardiograma semestralmente desde os quatro meses de idade e realizou exame citogenético que revelou cariótipo normal.

A mãe relatou atraso no desenvolvimento motor, como sentar sem apoio com um ano, engatinhar com um ano e três meses e andar com um ano e sete meses. Quanto ao desenvolvimento de linguagem, informou que a criança emitiu "tatá" aos dois anos e aos três anos e cinco meses não produzia palavras e frases, apresentava apenas algumas vocalizações e gestos, mas com compreensão oral normal.

\section{Dados de avaliação}

\section{Avaliação genética clínica e neurológica}

$\mathrm{Na}$ avaliação genética clínica, foi observada presença de globo ocular aumentado, fendas palpebrais alargadas, sobrancelhas arqueadas, distância intercantal aumentada, implantação discretamente baixa das orelhas, pescoço curto, estrabismo convergente, esclera azulada, mãos com digitais proeminentes, edema de membros inferiores, diâmetro ântero-posterior do tórax aumentado e sopro mesosistólico em foco mitral, retardo neuropsicomotor e de linguagem. Nessa época, recebeu o diagnóstico de síndrome de Kabuki. Na tomografia computadorizada, verificou-se massa hipodensa na região occipital.

\section{Avaliação otorrinolaringológica}

$\mathrm{Na}$ avaliação otorrinolaringológica, foi constatado histórico de otite e perfuração de membrana timpânica, além da presença de secreção em meato acústico externo, não sendo possível a realização do exame audiológico nessa ocasião.

\section{Avaliação audiológica}

Um ano após a avaliação otorrinolaringológica, foi realizada audiometria tonal condicionada por via aérea, que revelou limiares auditivos dentro do padrão de normalidade, de no mínimo 20dbNA em todas as frequências sonoras avaliadas.

\section{Avaliação fonoaudiológica clínica}

A avaliação fonoaudiológica clínica incluiu: análise de amostra de linguagem espontânea e dirigida, realizada por meio de observação comportamental e aplicação do Protocolo de Pragmática ${ }^{24}$, que investigou as iniciativas comunicativas, funções e meios comunicativos expressos. Além disso, foi avaliada a atividade lúdica por meio de observação comportamental para verificar o tipo de ação e manipulação dos objetos e interação com o terapeuta e com a mãe.

Verificou-se tempo de atenção auditiva e visual reduzida, sendo necessária a apresentação de diferentes estímulos sonoros e visuais em curtos períodos de tempo para manter a criança envolvida em atividades lúdicas.

Apresentou dificuldades de interação social, contato visual reduzido e desejava sair da sala por diversos momentos. Em algumas situações, apresentou intenção comunicativa, por meio de expressão facial, gestos indicativos e algumas vocalizações. Durante atividade lúdica utilizou com funcionalidade alguns objetos, tais como; mamadeira, cama, banheiro e sabonete, mas, na maioria das vezes, espalhava-os e batia no chão, sendo que suas ações e forma de manipulação de objetos não estavam compatíveis com a idade.

Quanto à emissão oral, demonstrou dificuldades na práxis oral e articulatória, não nomeou objetos ou figuras durante provas de nomeação e na atividade lúdica, emitindo apenas algumas vocalizações como "Aaaa", "Óóóó" e "Ai" para "Oi”. A recepção oral se mostrou aquém à idade, sendo capaz de compreender apenas ordens verbais simples de vocabulário cotidiano.

Para aplicação do Protocolo de Pragmática ${ }^{24}$, foram analisadas duas sessões de 30 minutos de filmagem, em que a criança apresentou, em média, cinco atos comunicativos por minuto, expressos preferencialmente pelo meio gestual e pelas funções comunicativas: Pedido de Objeto, Exibição, Protesto, Exploratória e Reativa.

Dessa forma, a criança apresentou alterações nos aspectos fonológicos, sintáticos, semânticos e pragmáticos da linguagem e recebeu diagnóstico fonoaudiológico de Distúrbio de Linguagem.

\section{RESULTADOS}

\section{Dados do processo terapêutico}

O processo terapêutico fonoaudiológico aqui exposto teve duração de três anos e iniciou-se logo após a avaliação fonoaudiológica, quando a criança 
apresentava três anos e seis meses de idade. As sessões individuais de terapia de linguagem ocorriam duas vezes por semana durante 50 minutos, sendo priorizada a interação entre terapeuta e paciente para promover o desenvolvimento cognitivo, a socialização e a comunicação da criança.

Os objetivos centrais da terapia fonoaudiológica foram aumentar a ocorrência de comportamentos comunicativos intencionais; favorecer a compreensão e o uso funcional da linguagem; e também oferecer orientações à família e à escola sobre como contribuir para o desenvolvimento da criança.

No início do processo terapêutico, a criança utilizava poucos gestos indicativos e vocalizações. Apresentava comportamentos agressivos e de birra com a terapeuta, algumas vezes sem motivo aparente, outras para atingir determinados objetivos, como sair da sala de terapia e receber atenção de outras crianças e terapeutas no corredor da clínica. Além disso, recusava-se a entrar na sala com a terapeuta, sendo necessária a presença da mãe durante as sessões.

Foram enfatizadas trocas comunicativas durante atividades lúdicas, tais como jogos de encaixe, massinha de modelar, brincadeiras de casinha, bonecas, atividades de vida diária, boliche, miniatura de animais, pintura com tinta guache e canetinhas, músicas infantis, instrumentos musicais, microfone e telefone, dentre outros. As situações de dramatização foram propostas com o objetivo de propiciar a representação simbólica, a interação social, além de levar a criança a se colocar em diferentes papéis. Durante essas atividades, foram interpretados os gestos e vocalizações da criança, considerando o contexto linguístico, social e físico.

No decorrer das sessões, as vocalizações passaram a ser usadas com maior frequência em relação aos gestos. Após cinco meses, a criança passou a apresentar maior número de atos comunicativos, por meio de expressão facial associada a emissões ininteligíveis e maior variedade de funções comunicativas mais interativas. Após um ano, a criança utilizava principalmente as funções comunicativas mais interativas, como: reconhecimento do outro, jogo compartilhado, pedido de ação e pedido de objeto e as menos interativas jogo, exploratória, protesto, expressão de protesto ${ }^{24}$. Houve melhora na interação social, passou a entrar sozinha com a terapeuta na sala em todas as sessões, embora ainda apresentasse problemas de comportamento, como agressividade e birra.

A partir do segundo ano de terapia, foi observada maior evolução quanto à emissão oral por meio de sons e palavras como "iii", "iaiá", "ai", "mamã", "papa", "tia". Notou-se também aumento da atenção compartilhada, da manipulação de objetos com funcionalidade e de esquemas simbólicos em suas brincadeiras.

Aos cinco anos e seis meses de idade, a criança apresentou uma crise convulsiva e foi internada na UTI por sete dias. Após esse episódio, foi observada regressão quanto ao comportamento linguístico e social da criança, com aumento dos comportamentos agressivos. Nesse período, foram necessárias sessões de terapia de linguagem em grupo e/ou com a participação da mãe para retomar o vínculo entre terapeuta e paciente.

As orientações aos pais foram realizadas com o objetivo de favorecer uma melhor interação com a criança, por meio de atividades lúdicas, conversas espontâneas e verbalização das ações da criança. Foram também necessárias orientações específicas relacionadas ao distúrbio de comportamento apresentado, que, segundo os pais, cotidianamente ocasionava situações desafiadoras e constrangedoras.

A terapeuta realizava ainda visitas à escola da paciente, oferecendo orientações sobre o processo terapêutico fonoaudiológico e o desenvolvimento de linguagem. Além disso, coletavam informações sobre o desempenho acadêmico, comunicação e interação social em ambiente escolar. Os professores relatavam agressividade em relação à professora e às outras crianças, dificuldades em propor atividades em sala de aula e desempenho escolar não compatível com sua idade cronológica.

Após três anos de terapia de linguagem, a criança apresentou melhora quanto aos aspectos semânticos e pragmáticos, como aumento da compreensão de ordens simples e complexas, expansão de vocabulário, gestos e expressões faciais, evolução quanto ao uso da linguagem e interação social, além de manutenção de atenção durante as atividades lúdicas. Quanto aos aspectos fonológicos e sintáticos, a evolução foi menos expressiva, como aumento da emissão oral por meio de vocábulos ininteligíveis e inteligíveis, com predominância no uso de vogais e algumas plosivas como /p/ e /b/, no entanto, não chegou a estruturar frases.

A conduta adotada até o momento foi a continuidade da terapia fonoaudiológica com ênfase nos aspectos semânticos e pragmáticos, por meio da atividade lúdica, visto que esta proposta tem se mostrado efetiva na intervenção do caso aqui descrito.

\section{DISCUSSÃO}

Os achados deste estudo confirmam dados da literatura referentes aos distúrbios de desenvolvimento associados à síndrome de Kabuki ${ }^{3,4,6}$, sendo que o caso aqui descrito apresentou características 
fenotípicas, como: alteração cardíaca congênita 25-27, icterícia neonatal, crises convulsivas, episódios de pneumonia, atraso no desenvolvimento motor ${ }^{5,6}$ e deficiência intelectual ${ }^{6}$.

A partir da avaliação fonoaudiológica da criança, observaram-se diversas manifestações clínicas citadas na literatura, como otite média secretora recorrente bilateral e perfuração de membrana timpânica ${ }^{6}$, além de alterações de motricidade oral ${ }^{18}$; dos aspectos fonológicos, sintáticos, semânticos e pragmáticos da linguagem $8,15,16,19,21$.

A alteração de linguagem mostrou-se significativa, assim como foi observado por outros autores 19. No entanto, também foram descritos casos com leve atraso no desenvolvimento de linguagem ${ }^{21}$. Em relação aos aspectos fonológicos e sintáticos, a criança comunicava-se quase que exclusivamente por emissões ininteligíveis e não estruturava frases ${ }^{7}$, também foram observadas dificuldades de compreensão oral e vocabulário restrito $8,14,15,19,21$, prejuízos na interação social e problemas de comportamento ${ }^{17}$, diferentemente de algumas crianças relatadas como comunicativas e sociáveis ${ }^{21}$.

Quanto à terapia de linguagem, verificou-se que foram alcançados os seus objetivos centrais. Assim, houve aumento da frequência de comportamentos comunicativos intencionais; melhora no uso funcional da linguagem e na compreensão oral; aumento de vocabulário; e utilização de meios comunicativos considerados mais efetivos durante a comunicação e consequentemente melhor interação e integração social.

Neste estudo, a terapia de linguagem focada em habilidades comunicativas e interacionais mostrou-se eficaz, principalmente quanto aos aspectos semânticos e pragmáticos da linguagem e quanto à interação social. No entanto, os aspectos sintáticos e fonológicos apresentaram apenas uma ligeira melhora em decorrência do importante prejuízo da praxia oral observado. Além disso, as orientações oferecidas aos familiares e à escola da criança foram fundamentais para a eficácia da intervenção fonoaudiológica. A literatura descreveu casos de síndrome de Kabuki que apresentaram melhora quanto aos aspectos fonéticos e fonológicos, sem evolução na prosódia e apontou a necessidade de intervenção para melhor desenvolver fala e linguagem de indivíduos com a síndrome ${ }^{19}$.

Contudo, é importante considerar outros fatores que podem ter tido implicações no desenvolvimento da comunicação da criança em questão, como a deficiência intelectual, otite recorrente, episódios de crise convulsiva e internações, além da dinâmica familiar e escolar em que está inserida. A relevância de se considerar esses aspectos também foi ressaltada por outros autores ${ }^{16}$.

Estudos sobre o prognóstico de desenvolvimento da linguagem na síndrome de Kabuki são escassos. No entanto, devido à heterogeneidade clínica descrita pela literatura 8,15,19,21, é provável que o prognóstico fonoaudiológico de indivíduos com a síndrome seja bastante variável. Assim, é importante a contribuição de estudos sobre as características de comunicação nesses casos, a fim de auxiliar o diagnóstico e o planejamento terapêutico o mais precocemente possível, para promover melhor qualidade de vida da criança e sua família.

\section{CONCLUSÃO}

Este estudo permitiu divulgar o percurso de atuação fonoaudiológica na síndrome de Kabuki, do processo diagnóstico à terapia de linguagem. A partir da avaliação fonoaudiológica, foi possível constatar o fenótipo desta síndrome, identificando a presença de déficit cognitivo e distúrbio de linguagem, associado a problemas comportamentais. Assim, destaca-se a necessidade da participação de fonoaudiólogos junto a equipes multidisciplinares nesses casos, a fim de possibilitar a realização de diagnóstico e intervenção o mais precocemente possível.

No que se refere à terapia de linguagem, foi priorizada a interação entre terapeuta e paciente para promover o desenvolvimento cognitivo, social e linguístico da criança. Foi observada melhora quanto aos aspectos semânticos e pragmáticos, como aumento da compreensão oral, expansão de vocabulário, gestos e expressões faciais, evolução quanto ao uso da linguagem e à interação social. Quanto aos aspectos fonológicos e sintáticos, a evolução foi menos expressiva em decorrência do prejuízo na praxia oral.

Além disso, foram oferecidas orientações que permitiram a troca de informações entre família, escola e terapeuta, que se mostraram fundamentais no planejamento de estratégias terapêuticas mais adequadas à realidade da criança.

Vale ressaltar o reduzido número de estudos sobre os aspectos fonoaudiológicos na SK, principalmente no que se refere ao processo terapêutico e sua evolução. Dessa forma, estes dados podem contribuir para elucidar lacunas existentes na literatura científica e para subsidiar intervenções clínicas nesses casos. 


\begin{abstract}
Background: this study aimed at describing the language intervention process in a Kabuki syndrome case, emphasizing the language therapy. Procedures: this is a report of a six and a half year-old girl, diagnosed with Kabuki syndrome, who attended language therapy for three years at a clinical school. The documental analysis of the medical register form was made, concerning interview evaluation and language therapeutic reports, examination and multidisciplinary evaluations. When the child was three years and five months old, she went through language evaluation and showed typical characteristics of Kabuki syndrome: cardiac alterations, pneumonia, recurrent secretory otitis media, orthopedic alterations and convulsion crises. For the language evaluation, she presented a reduced period of hearing and visual attention, impaired oral comprehension, communication through only a few gestures and sounds and also behavioral problems. Results: the therapeutic process aimed to promote language development emphasizing social interaction through ludic activities, and a familiar and scholar follow-up. An improvement was observed for comprehension and oral language, social interaction and attention steadiness. Conclusion: this study allowed the trajectory disclosure of language development concerning the Kabuki syndrome, from the diagnosis to the language therapy. It was observed that the therapy was effective concerning communication abilities and social interaction. These data may contribute to clarify some aspects in the literature towards the language therapy for the cited syndrome and also suggest clinical interventions for these cases.
\end{abstract}

KEYWORDS: Genetics; Language; Evaluation; Rehabilitation

\section{REFERÊNCIAS}

1. Cuscó I, del Campo M, Vilardell M, González E, Gener B, Galán E, et al. Array-CGH in patients with Kabuki-like phenotype: identification of two patients with complex rearrangements including 2q37 deletions and no other recurrent aberration. BMC Medical Genetics. 2008; 9(1):27.

2. Hou JW. Variable expressivity in a family with Kabuki make-up (Niikawa-Kuroki) syndrome. Chang Gung Med J. 2004; 27(4):307-11.

3. Engelen JJ, Loneus WH, Vaes-Peeters G, Schrander-Stumpel CT. Kabuki syndrome is not caused by an $8 p$ duplication: a cytogenetic study in 20 patients. Am J Med Genet A. 2005; 132A(3):276-7.

4. Schoumans J, Nordgren A, Ruivenkamp C, Brondum-Nielsen K, Teh BT, Annéren G, et al. Genome-wide screening using array-CGH does not reveal microdeletions/ microduplications in children with Kabuki syndrome. Eur J Hum Genet. 2005; 13(2):260-3.

5. Salpietro DC, Guarneri F, Rigoli L, Bruglia S, Guarneri C, Vaccaro M. What syndrome is this? Kabuki make-up syndrome. Pediatr Dermatol. 2007; 24(3):309-12.

6. Niikawa N, Matsuura N, Fukushima Y, Ohsawa T, Kajii T. Kabuki make-up syndrome: a syndrome of mental retardation, unusual facies, large and protruding ears, and postnatal growth deficiency. J Pediatr. 1981; 99(4):565-9.
7. Souza JC, Ribeiro TCC, Ribeiro RC. A síndrome da máscara do Cabúqui. J Pediatr. 1996; 72(5):341-4.

8. Defloor T, van Borsel J, Schrander-Stumpel CT, Curfs LM. Expressive language in children with Kabuki syndrome. Am J Med Genet A. 2005; 132A(3):256-9.

9. Milunsky JM, Huang XL. Unmasking Kabuki syndrome: chromosome 8p22-8p23.1 duplication revealed by comparative genomic hybridization and BAC-FISH. Clin Genet. 2003; 64(6):509-16.

10. Kuniba H, Tsuda M, Nakashima M, Miura $\mathrm{S}$, Miyake N, Kondoh T, et al. Lack of C20orf133 and FLRT3 mutations in 43 patients with Kabuki syndrome in Japan. J Med Genet. 2008; 45(7):479-80.

11. Miyake N, Shimokawa O, Harada N, Sosonkina $\mathrm{N}$, Okubo $\mathrm{A}$, Kawara $\mathrm{H}$, et al. No detectable genomic aberrations by BAC array CGH in Kabuki make-up syndrome patients. Am J Med Genet A. 2006; 140(3):291-3.

12. Milunsky JM, Maher TA, Zhao G, Huang $X L$, Wang Z, Zou Y. A re-examination of the chromosome 8p22-8p23.1 region in Kabuki syndrome. Clin Genet. 2008; 73(5):502-3.

13. Santos AS, Bárbara BF, Pimentel HM, Sousa OM, Castro NP. Aspectos otorrinolaringológicos da Síndrome de Kabuki. ACTA ORL. 2008; 26(1):60-2.

14. Gabrieli APT, Rovaris FV, Bisol LE, Borges L, Michelin MM, Lovatto L. Síndrome da maquiagem de Kabuki. Acta Ortop Bras. 2002; 10(3):57-61. 
15. Mervis $C B$, Becerra AM, Rowe ML, Hersh $\mathrm{JH}$, Morris CA. Intellectual abilities and adaptive behavior of children and adolescents with Kabuki syndrome: a preliminary study. Am J Med Genet A. 2005; 132A(3):248-55.

16. Van Lierde KM, Van Borsel J, Van Cauwenberge $P$. Speech patterns in Kabuki make-up syndrome: a case report. J Commun Disord. 2000; 33(6):447-61. 17. Sari BA, Karaer K, Bodur S, Soysal AS. Case report: autistic disorder in Kabuki syndrome. J Autism Develop Disord. 2008; 38(1):198-201.

18. Takada K, Fukushima $\mathrm{H}$, Watanabe $\mathrm{S}$, Ishida $\mathrm{M}$, Ogasawara $\mathrm{H}$, Motokawa W. Occlusal guidance of two Kabuki make-up syndrome patients: case reports. J Clin Pediatr Dent. 2004; 28(2):113-8.

19. Burke LW, Jones MC. Kabuki syndrome: underdiagnosed recognizable pattern in cleft palate patients. Cleft Palate Craniofac J. 1995; 32(1):77-84. 20. Muniz MCMC, Magalhães Júnior HV. Distúrbios da comunicação na síndrome de Kabuki: estudo de um caso. Pró-Fono. 2008; 20(Supl):7-10.

21. Ilyina $H$, Lurie I, Naumtchik I, Amoashy D, Stephanenko G, Fedotov V, et al. Kabuki make-up (Niikawa-Kuroki) syndrome in the Byelorussian register of congenital malformations: ten new observations. Am J Med Genet. 1995; 56(2):127-31.
22. Adam MP, Hudgins L. Kabuki syndrome: a review. Clin Genet. 2005; 67(3):209-19.

23. White SM, Thompson EM, Kidd A, Savarirayan R, Turner A, Amor D, et al. Growth, behavior, and clinical findings in 27 patients with Kabuki (NiikawaKuroki) syndrome. Am J Med Genet A. 2004; 127A(2):118-27.

24. Fernandes FDM. Pragmática. In: Andrade CRF, Befi-Lopes DM, Fernandes FDM, Wertzner HF. ABFW: Teste de linguagem infantil nas áreas de fonologia, vocabulário, fluência e pragmática. Carapicuiba: Pró-Fono; 2004. p. 83-97.

25. Shahdadpuri R, Lynch SA, Murchan H, McMahon CJ. A novel constellation of cardiac findings for Kabuki syndrome: hypoplastic left heart syndrome and partial anomalous pulmonary venous drainage. Pediatr Cardiol. 2008; 29(4):820-2.

26. Moral S, Zuccarino F, Loma-Osorio P. Double aortic arch: an unreported anomaly with Kabuki syndrome. Pediatr Cardiol. 2009; 30(1):82-4.

27. Schertler T, Wildermuth S, Teodorovic N, Mayer D, Marincek B, Boehm T. Visualization of congenital thoracic vascular anomalies using multi-detector row computed tomography and two- and threedimensional post-processing. Eur J Radiol. 2007; 61(1):97-119.

DOI: 10.1590/S1516-18462010005000045

RECEBIDO EM: 22/05/2009

ACEITO EM: 09/09/2009

Endereço para correspondência:

Andréa Regina Nunes Misquiatti

Av. Hygino Muzzi Filho, 737 Caixa Postal: 181

Marília - SP

CEP: 17525-050

Email: amisquiatti@uol.com.br 